\title{
Investigating Cervical Flexion-Relaxation Ratio in Patients with Cervical Lateral Spinal Stenosis Compared with Asymptomatic Controls: a case control study
}

\section{Zahra Akbarnejad-Basra}

Master of physical therapy, School of Rehabilitation, Babol University of Medical Sciences

Khodabakhsh Javanshir

Associate Professor of Physical Therapy, Department of Physical Therapy, School of Rehabilitation Mobility Impairment Research Center, Health Research Institute, Babol University of Medical Sciences

\section{Payam Saadat}

Associate Professor of Neurology, Mobility Impairment Research Center, Health Research Institute, Babol University of Medical Sciences

Roghayeh Mousavi-Khatir ( $\nabla$ rmosavi_pt@yahoo.com )

Assistant Professor of Physical Therapy, Department of Physical Therapy, School of Rehabilitation, Mobility Impairment Research Center, Health Research Institute

\section{Research Article}

Keywords: flexion-relaxation ratio, FRR, cervical erector spinae, lateral spinal stenosis, surface electromyography

Posted Date: February 9th, 2022

DOI: https://doi.org/10.21203/rs.3.rs-1299831/v1

License: (a) This work is licensed under a Creative Commons Attribution 4.0 International License. Read Full License 


\section{Abstract \\ Background}

The cervical flexion-relaxation ratio (FRR) is known as a quantitative measure of the ability of the cervical extensor muscles to relax during the full flexion of the cervical spine. In this sense, the variations in the cervical FRR indicate some changes in the neuromuscular pattern of extensor muscles, and consequently, their functions. Compared with patients suffering from non-specific neck pain, the changes occurring in the pattern of the neck muscles' functions in cases with cervical spinal stenosis are not yet well understood. Therefore, this study aimed to compare cervical FRR in patients with cervical lateral spinal stenosis and asymptomatic controls.

\section{Methods}

In total, 25 patients with cervical lateral spinal stenosis (aged $44.7 \pm 10.4$ ) and 25 asymptomatic individuals (aged $44.3 \pm 10.8$ ) were included in this study. These participants performed full-neck flexion, and at the same time, the electrical activity of the cervical erector spinae (CES) muscles was recorded bilaterally. Cervical FRR was further calculated by analyzing the electromyographic (EMG) data recorded for each muscle.

\section{Results}

The mean cervical FRR was significantly lower $(\mathrm{P}<0.05)$ and the FRR asymmetry $(\Delta F R R)$ was significantly higher $(P=0.003)$ in the patients than asymptomatic controls. In addition, the cervical FRR of the involved side was significantly lower in the patients compared with that in the non-involved one $(P=0.004)$. There was also a significant difference in the cervical extension (CE) angle between both study groups $(P<0.001)$, while the cervical flexion $(C F)$ angle was not different $(P>0.05)$.

\section{Conclusion}

According to the study results, it was concluded that the neuromuscular pattern of the cervical muscles could change asymmetrically and the $\mathrm{CE}$ angle could decline in individuals with cervical lateral spinal stenosis. Therefore, such patients will be more susceptible to biomechanical changes and persistent pain if the cervical muscles are not considered in their treatment.

\section{Background}

Spinal stenosis refers to a condition characterized by the narrowing of the spinal canal or vertebral foramen that compresses the spinal cord or the nerve root, resulting in pain and some neurological symptoms $(1,2)$. As retrieved from the related literature, the degenerative form of cervical spinal stenosis, 
i.e., cervical spondylotic myelopathy (CSM), is much more prevalent in the elderly population $(1,3,4)$. Based on its anatomical location, spinal stenosis is grouped into two categories where the spinal cord is damaged, namely, central and lateral/foraminal stenosis $(1,5,6)$. The signs and symptoms of spinal stenosis depend on the spinal cord or nerve root compression severity and involvement $(3,6)$. In general, patients with cervical spinal stenosis may complain of pain in the neck and even experience unilateral or bilateral radicular pain in the upper extremities, as well as numbness and weakness during the fine movements of their limbs, and possibly gait disorders, including imbalance $(1,3)$. At present, magnetic resonance imaging (MRI) is a valuable and common non-invasive method for diagnosing spinal stenosis compared to other technologies $(3,5,7)$; however, the severity of patients' clinical symptoms may not sometimes match that of spinal stenosis reported on MRI, as confirmed in some studies $(4,8,10)$. Depending on the symptoms, conservative or surgical treatment is often chosen to improve patient's condition $(1,3)$. In this sense, conservative treatment consists of medication use, medical support, and physiotherapy (1). Considering the nerve nutrition of the muscles surrounding the cervical spine, spinal stenosis in this area may lead to denervation, and consequently, increase fat infiltration into these muscles $(8,11)$. On the other hand, fear of movement due to the exacerbation of stenosis and pain $(1,6)$ augments the risk of cervical muscle disuse and atrophy in such cases (12). Although previous studies have thus far reported the morphology of paraspinal muscle changes in patients with lumbar and cervical spinal stenosis $(8,11,13,14)$, evaluating neck muscle function, based on electrical activity, in patients with spinal stenosis seems to be essential with regard to the key role of neck muscles in providing the stability of this area.

The flexion-relaxation phenomenon (FRP) here means the relaxation of the superficial extensor muscles following the flexion of the spine; in other words, the electromyographic (EMG) activity of the superficial erector spinae (ES) muscles diminishes or switches off once the spine is fully flexed, referred to as FRP $(15,17)$. In fact, this phenomenon emerges due to the transfer of the kinetic force torque to the passive elements of the spine $(15,17,18)$. In this line, Pialasse et al. had confirmed the existence of flexionrelaxation in the cervical spine (15). Mousavi-Khatir et al. had further observed FRP change parameters after 10 minutes of sustained full cervical flexion (CF) in healthy individuals (19). According to the related literature, flexion-relaxation ratio (FRR) is known as a quantitative measure for calculating $\operatorname{FRP}(17,20$, 22), and even a reliable method to examine neuromuscular dysfunction, as suggested by Murphy et al. (16). Moreover, Yeo et al. had reported that FRR could mostly vary in individuals with forward head posture compared with healthy controls (22). However, few studies have so far shown that FRP in patients with neck pain has changed $(16,17)$. Comparing FRR between healthy cases and patients with chronic neck pain, Murphy et al. had further noted that FRR in patients had been significantly higher than that in healthy individuals (16). In another study, Maroufi et al. had found FRP in only $36.3 \%$ of patients with chronic neck pain, and suggested that neck muscle activity could vary in the majority of motor tasks (17). Considering that patients with spinal stenosis need to maintain spinal flexion to prevent pain $(1,6)$, it seems that the study of superficial ES muscle activity pattern and FRP in patients with neck canal stenosis is of utmost importance. However, no study was found, to the best of the authors' knowledge, 
investigating the electrical activity of the superficial cervical ES muscles in patients with cervical lateral spinal stenosis.

In addition to FRR, two other features were examined in this study; the first one was FRR asymmetry ( $\triangle F R R$ ), which was compared due to the one-sidedness of patients' symptoms, and the second feature was the CF and cervical extension (CE) range of motion (ROM), which could often show a discrepancy in neck disorders (23). Therefore, the primary objective of this study was to evaluate mean FRR in patients with cervical lateral spinal stenosis and compare it with asymptomatic controls, and the secondary objective was to compare $\triangle \mathrm{FRR}$ in ES muscles on both sides between patients and controls. As the tertiary objective, the present study aimed to compare the CF and CE angles between both groups.

\section{Methods}

This observational case-control study was performed in the Biomechanics Laboratory of the Rehabilitation School affiliated to Babol University of Medical Sciences, Babol, Iran. The ethical approval of this study was also granted by the Research Ethics Committee of Babol University of Medical Sciences, Babol, Iran (IR.MUBABOL.REC.1399.245).

\section{Participants}

In total, 25 patients ( 5 men and 20 women) with cervical lateral spinal stenosis and 25 asymptomatic controls ( 5 men and 20 women) participated in this study. The patients were referred to the Biomechanics Laboratory of a neurology clinic affiliated to Babol University of Medical Sciences, Babol, Iran, and were included in the study after physical evaluations by a skilled physiotherapist, having 15-year experience in the field of cervical musculoskeletal disorders, to investigate the interference of other musculoskeletal disorders in the neck and shoulder regions. The patients were accordingly selected for this study if they had a confirmed MRI report of cervical lateral spinal stenosis, pain in the neck or one upper extremity, and no history of cervical spine surgery. The patients were also excluded if they were candidates for surgery to treat stenosis, or had a history of neck rehabilitation and therapy over the past three months, or the evidence of a tumor, a fracture, or an infection in the cervical spine. The participants in the control group were further recruited from individuals with no history of pain and disorder in the neck and the upper extremity in the last year. The ones with a history of neck surgery, regular exercise, and unwillingness to cooperate were consequently excluded from the study. All the participants received explanations about the testing process, signed an informed consent form, and completed a personal information questionnaire.

\section{Experimental Protocol}

All the participants were tested in one session for 40 minutes. They were first seated in a chair with a back while the soles of their feet were on the ground and the knees and hips were at a $90^{\circ}$ angle. Then, their thoracic region was fixed to the chair with a strap, at the level of the scapula, holding the arms comfortably at the side of the body. The participants were next asked to look at the target at the eye level 
during the test and keep their head and neck in a neutral position, determined as the midpoint between full neck flexion and extension (19) by an experienced physiotherapist. Accordingly, the participants were requested to perform the flexion and extension of the cervical spine to the end of the range, and the midpoint was targeted as the neutral position of the head for each individual (19). In addition, they were asked to flex and extend their neck three times from the midpoint as far as they could in order to assess the cervical ROM on the sagittal plate. The average of three repetitions in each movement was further considered as the ROM of flexion and extension. Afterward, the participants performed the following steps, respectively: (1) keeping the head in the starting position for 4 seconds, (2) bending the head until the chin was close to the upper chest, and this was done in 4 seconds, (3) maintaining this position for 4 seconds, and (4) returning (re-extending) the head to its original position within 4 seconds. An audio signal was also applied to determine the speed of movement in each step (16). Before the performance, all the test steps were explained to the participants and they were allowed to practice the test steps several times. Of note, each participant repeated the testing process three times, and an interval was provided to prevent muscle fatigue for two minutes between each time.

\section{Instrumentation}

The EMG signals were recorded bilaterally from the cervical ES, using a surface EMG device, DataLITE (PIONEER, Biometrics Ltd., UK). The wireless bipolar Ag/AgCl surface electrodes were then bilaterally attached on skin, $2 \mathrm{~cm}$ outside the center of the spinous process of the fourth cervical vertebra, in line with the ES muscle fibers $(19,24)$. The inter-electrode distance was equal to $20 \mathrm{~mm}$. Before the electrode placement, the examiner prepared the participant's skin by light abrasion, swabbing with alcohol, and shaving if it was necessary.

The sampling rate was $1000 \mathrm{~Hz}$ with a band-pass filter at 20-480 $\mathrm{Hz}$ that was amplified with a common mode rejection ratio $>110 \mathrm{~dB}$, an overall gain of 1,000 , and noise $<1 \mu \mathrm{V}$ root mean square (RMS). To determine the exact test steps, the neck flexion and extension angles were recorded using an electrogoniometer (the sampling rate of $1000 \mathrm{~Hz}$, Biometrics, Ltd., UK) synchronized with the EMG data.

\section{Data Analysis}

The raw EMG waves were filtered and their RMS was detected by the Biometrics Analysis Software for DataLITE, in 50 ms Time Windows. FRR was then calculated as an indicator of the presence of FRP by dividing the maximum RMS in the cervical re-extension stage (viz. the fourth stage) by the average RMS in the stage of maintaining full flexion (i.e., the third stage). Afterward, the mean FRR of both (left and right) sides was utilized for statistical analysis (19). The difference in muscle FRR on both sides actually signified asymmetry in the FRP presentation. For this purpose, $\Delta F R R$ was calculated as follows: $\Delta F=$ |right FRR - left FRR| (20). The FRR index was further calculated for cervical ES on both sides. In addition, the amount of FRR on the involved side was compared with the non-involved one in the patient group during an intra-group comparison.

\section{Statistical Analysis}


The SPSS (ver. 20) Statistics software package (SPSS Inc., Chicago, USA) was used for data analysis purposes. Independent-samples t-test was also applied to compare the distribution of the demographic characteristics between both groups of the participants. As well, independent-samples t-test was recruited to compare the mean FRR, $\triangle \mathrm{FRR}$, and $\mathrm{CF}$ and $\mathrm{CE}$ angles between the two groups. Paired-samples t-test was additionally employed to compare FRR between the involved and non-involved sides in patients. The statistically significant level in all tests was considered as $\mathrm{P}<0.05$.

\section{Results}

Before analyzing the study results, the normality of the data was confirmed in all research variables by the Kolmogorov-Smirnov test, but it was not significant at the level of 0.05 . In terms of the demographic variables, there were no significant differences in the mean age, weight, height, and body mass index (BMI) $(P>0.05)$. Moreover, all the study participants were right-handed. The results are presented in Table 1.

The study results revealed that the mean FRR in the patients was significantly lower than that in the controls $(P=0.015)$ (Figure 1). To compare the asymmetry between FRR in the right and left cervical erector spinae (CES), $\triangle$ FRR was compared and the findings established that the asymmetry in the patients was significantly higher than that in the controls ( $P=0.003)$. The CE.ROM in the patients was significantly lower than that in the controls $(P<0.001)$, while the CF.ROM angle was not significantly different between the two groups $(P=0.25)$ (Figure 1). Table 2 illustrates the information related to these data.

In total, 17 and 8 participants in the patient group had right- and left-sided involvement, respectively. Likewise, a significant decrease was observed in the FRR of the involved side compared with the noninvolved one in the patients $(P=0.004)$ (Table 3$)$.

\section{Discussion}

The present study examined the cervical CES FRR in patients with cervical lateral spinal stenosis compared with asymptomatic controls.

Of note, FRR is a quantitative measure for evaluating FRP in ES muscles as well as a practical and reliable method for reflecting on the functional pattern of these muscles in patients with neck pain (16). Accordingly, the study results showed that cervical FRR in patients with cervical lateral spinal stenosis was significantly lower than that in asymptomatic controls, which was consistent with the findings of previous studies on neck pain $(16,17,25)$. Murphy et al. had further observed a significant drop in mean FRR in patients with chronic neck pain compared with controls, and had reported the high reliability of the results and differences between groups, suggesting that FRR was a good indicator of the changes in neuromuscular function in patients with neck pain (16). Shamsi et al., comparing the FRR of the CES muscles and the upper trapezius of patients with non-specific neck pain and healthy individuals, had 
similarly observed that the FRR in patients was lower than that in healthy individuals, but there was no significant difference in the upper trapezius muscles (25). In fact, the fall in FRR may occur due to the elevated activity of ES muscles in the state of full flexion or a rise in the activity of these muscles during re-extension of the full flexion. According to Maroufi et al., boosting the activity of the CES muscles in the full flexion phase could have a significant effect on reducing FRR in patients with chronic neck pain compared with controls (17). Decreased FRR in patients with cervical stenosis in this study may also indicate the changes in neuromuscular function and muscle activity pattern. One possible hypothesis to explain the reduced FRR in these patients is no relaxation of the cervical superficial ES muscles in the full flexion phase, attributed to the increased activity of these muscles to stabilize the neck following a decline in the deep contractile units of muscle fiber after increased fat infiltration in the state of nerve root compression $(14,26)$. To the best of the authors' knowledge, this study was one of the first attempts to investigate FRR in patients with cervical lateral spinal stenosis, so further research is needed to evaluate other features of FRP.

As recommended in the study by Dulcina et al., $\triangle F R R$ was used to compare the asymmetry of the cervical FRR between the two groups (20) and the results showed that the asymmetry of CES FRR in patients with cervical lateral spinal stenosis was significantly higher than that in asymptomatic controls. Dulcina et al. had similarly measured asymmetric FRR in the ES muscles and superficial lumbar multifidus in patients with non-specific low back pain, and had observed that the $\triangle F F R$ of the ES muscles in patients was higher than that in controls, and stated that asymmetric FRR could cause unilateral overactivity of the lumbar ES (20). Previous studies had also reported muscle atrophy associated with cervical myelopathy and radiculopathy $(11,13,14,27,28)$. Besides, Fortin et al. had reported more asymmetry in the crosssectional area of the paraspinal muscles below the compression level in patients with degenerative cervical myelopathy, and had reported that elevated asymmetry was associated with reduced functional scales (29). Therefore, the changes in muscle morphology could shape muscle function in patients with nerve compression syndrome in the cervical region $(14,29)$. In other words, the asymmetry in atrophy can be associated with that in muscle activity since muscle atrophy is related to a reduction in the basic contractile units of muscle fiber. In addition to the inter-group comparison, an intra-group comparison was also performed in the patient group between the FRR of the involved and non-involved sides, and it was revealed that the FRR of the involved side was significantly lower than that of the non-involved one. In line with the study results, Shamsi et al. had reported significant differences between FRR on the left and right sides in a group with non-specific neck pain, and had suggested that asymmetric FRR could induce unilateral overactivity of the CES muscle, and consequently, persistent pain (25). In another study, Park et al. had demonstrated that the pattern of muscle activity on the painful side could change more in patients with unilateral posterior neck pain, and the asymmetry in pain could affect that of muscle activity (30). An imbalance in bilateral paracervical muscle activity had been further reported during neck endurance testing in patients with cervical radiculopathy (31). Therefore, the existence of asymmetry in the pattern of muscle activity and FRR are made possible due to the one-sided symptoms and pain in the patients participating in this study. However, it is not yet clear whether this asymmetry comes from the 
effect of unilateral symptoms or it is the cause. In general, it may be effective in perpetuating or reversing pain due to unilateral muscle overactivity.

The neck ROM, as one of the components studied in patients with neck pain $(32,33)$, was also examined in this study, and the results showed that the neck extension ROM was significantly lower in patients with cervical lateral spinal stenosis than that in the controls. Other studies had further reported significant differences in the cervical total ROM of the sagittal plate in patients with cervical myelopathy $(34,35)$. The changes in the neck ROM can be thus assumed as one of the disruptive factors in neck biomechanics. In this respect, Yeo et al. had detected a descending trend in FRR in a group with forward head posture, and had concluded that fatigue in the ES muscles may be a factor in changing FRR (22). Mousavi-Khatir et al. had also studied the effect of static neck flexion on FRP in healthy individuals, and had observed that the FRP parameters such as FRR, neck flexion angle, and amplitude of ES muscle activity had changed after 10 minutes of keeping the head in the flexion position (19). Of note, patients with spinal stenosis avoid extension movements to prevent pain, irritation, and exacerbation of symptoms, and typically tend to maintain the flexion posture $(1,8)$. As suggested by Mousavi-Khatir et al., maintaining neck flexion in the long run may change the length-tension characteristics, reduce the stiffness of passive viscoelastic tissues, and ultimately give rise to compensatory cervical muscle activity (19). Accordingly, it can be assumed that the changes in the neck ROM in patients with cervical lateral spinal stenosis can be a factor in changing their muscle activity pattern.

This study had several limitations. Although there was no significant difference between the contextual variables, due to the attrition in the number of volunteers for their reluctance to participate in the study following the pandemic, coronavirus disease 2019, some interfering conditions such as occupation and socioeconomic status of the participants were not taken into account. As well, only patients with mild-tomoderate spinal stenosis were evaluated in this study, and the cases with severe spinal stenosis were not included for ethical considerations. Therefore, more studies are needed to clarify the relationship between spinal stenosis severity and FRR.

The study findings also revealed that FRR and its asymmetry were different in patients with cervical lateral spinal stenosis compared with those in asymptomatic individuals, indicating the changes in FRP in these patients, so further research is required to investigate other features of FRP as well as the presence or absence of FRP in patients with cervical lateral spinal stenosis. In addition, knowing about the changes in the pattern of cervical muscle function in patients with cervical spinal stenosis is effective in planning rehabilitation practices and treatments to maintain the correct function pattern of the muscles in this area and prevent pain reversibility, along with other procedures to relieve pressure on the spinal cord and the nerve roots. Therefore, more studies are needed to determine the relationship between cervical spinal stenosis, the changes in muscle activity pattern, and the effect of rehabilitation exercises on it in patients with cervical lateral spinal stenosis.

\section{Conclusion}


The present study showed that FRR in the patients with lateral spinal stenosis was significantly different from that in asymptomatic controls, and even more asymmetry was observed in the CES FRR of the patients with lateral spinal stenosis. In addition, a significant difference in the CE.ROM in the patients with cervical lateral spinal stenosis may affect the biomechanics of the cervical region in these patients. Therefore, more research is needed to address this issue. Furthermore, it is suggested to investigate the relationship between cervical ROM, spinal stenosis severity, and functional status of patients with FRR changes, as well as the presence or absence of FRP and the variations in muscle activity pattern in patients with lateral spinal stenosis in future studies (14).

\section{Abbreviations}

\section{BMI}

Body mass index

CE

Cervical extension

CES

Cervical erector spinae

CF

Cervical Flexion

CSM

Cervical spondylotic myelopathy

\section{EMG}

Electromyography

ER

Erector spinae

FRR

Flexion relaxation ratio MRI:Magnetic resonance imaging

\section{ROM}

Range of motion

\section{Declarations}

Acknowledgements: We would like to express our thanks to all the staff of Babol University Medical Science, school of Rehabilitation as well as to all individuals helping us in completing this research project.

\section{Authors' contributions:}

ZA participated in the concept and design of the study, performed data acquisition, analysis and interpretation and drafted the manuscript. RM participated in the concept and design of the study, performed data acquisition, analysis and interpretation and was a major contributor in writing the 
manuscript. KJ participated in the concept and design of the study. PS participated in the collection of the experimental group. All authors read and approved the final manuscript.

Funding: No funding was received.

\section{Availability of data and materials}

The datasets generated and/or analyzed during the current study are not publicly available due to restrictions e.g. their containing information that could compromise the privacy of research participants, but are available from the corresponding author on reasonable request

\section{Ethics approval and consent to participate:}

The ethical approval of this study was also granted by the Research Ethics Committee of Babol University of Medical Sciences, Babol, Iran (IR.MUBABOL.REC.1399.245). All the participants received explanations about the testing process, signed an informed consent form, and completed a personal information questionnaire. Our study was conducted in accordance with the Declaration of Helsinki.

Consent for publication: Not applicable.

Competing interests: The authors declare that they have no competing interests.

\section{Author's details}

1: Master of physical therapy, School of Rehabilitation, Babol University of Medical Sciences, Babol, Iran

2: Associate Professor of Physical Therapy, Department of Physical Therapy, School of Rehabilitation Mobility Impairment Research Center, Health Research Institute, Babol University of Medical Sciences, Babol, Iran

3: Associate Professor of Neurology, Mobility Impairment Research Center, Health Research Institute, Babol University of Medical Sciences, Babol, Iran

4: Assistant Professor of Physical Therapy, Department of Physical Therapy, School of Rehabilitation, Mobility Impairment Research Center, Health Research Institute, Babol University of Medical Sciences, Babol, Iran

\section{References}

1. Melancia JL, Francisco AF, Antunes JL. Spinal stenosis. Handbook of clinical neurology. 2014;119:541-9.

2. Tong Y, Huang Z, Hu C, Fan Z, Bian F, Yang F, et al. A comparison study of posterior cervical percutaneous endoscopic ventral bony decompression and simple dorsal decompression treatment 
in cervical spondylotic radiculopathy caused by cervical foraminal and/or lateral spinal stenosis: a clinical retrospective study. BMC musculoskeletal disorders. 2020;21:1-10.

3. Tracy JA, Bartleson J. Cervical spondylotic myelopathy. The neurologist. 2010;16(3):176-87.

4. Kang Y, Lee JW, Koh YH, Hur S, Kim SJ, Chai JW, et al. New MRI grading system for the cervical canal stenosis. American Journal of Roentgenology. 2011;197(1):W134-W40.

5. Barzin M. MRI Findings in Patients with Spinal Canal Stenosis. Journal of Guilan University of Medical Sciences. 2011;20(79):40-8.

6. Lee SY, Kim T-H, Oh JK, Lee SJ, Park MS. Lumbar stenosis: a recent update by review of literature. Asian spine journal. 2015;9(5):818.

7. Adamova B, Bednarik J, Andrasinova T, Kovalova I, Kopacik R, Jabornik M, et al. Does lumbar spinal stenosis increase the risk of spondylotic cervical spinal cord compression? European Spine Journal. 2015;24(12):2946-53.

8. Yarjanian JA, Fetzer A, Yamakawa KS, Tong HC, Smuck M, Haig A. Correlation of paraspinal atrophy and denervation in back pain and spinal stenosis relative to asymptomatic controls. PM\&R. 2013;5(1):39-44.

9. Fortin M, Lazáry À, Varga PP, Battié MC. Association between paraspinal muscle morphology, clinical symptoms and functional status in patients with lumbar spinal stenosis. European Spine Journal. 2017;26(10):2543-51.

10. Haig AJ, Geisser ME, Tong HC, Yamakawa KS, Quint DJ, Hoff JT, et al. Electromyographic and magnetic resonance imaging to predict lumbar stenosis, low-back pain, and no back symptoms. JBJS. 2007;89(2):358-66.

11. Cloney M, Smith AC, Coffey T, Paliwal M, Dhaher Y, Parrish T, et al. Fatty infiltration of the cervical multifidus musculature and their clinical correlates in spondylotic myelopathy. Journal of Clinical Neuroscience. 2018;57:208-13.

12. Lindstrøm R, Schomacher J, Farina D, Rechter L, Falla D. Association between neck muscle coactivation, pain, and strength in women with neck pain. Manual therapy. 2011;16(1):80-6.

13. Hou X, Lu S, Wang B, Kong C, Hu H. Morphologic Characteristics of the Deep Cervical Paraspinal Muscles in Patients with Single-Level Cervical Spondylotic Myelopathy. World neurosurgery. 2020;134:e166-e71.

14. Fortin M, Wilk N, Dobrescu O, Martel P, Santaguida C, Weber MH. Relationship between cervical muscle morphology evaluated by MRI, cervical muscle strength and functional outcomes in patients with degenerative cervical myelopathy. Musculoskeletal Science and Practice. 2018;38:1-7.

15. Pialasse J-P, Dubois J-D, Choquette M-HP, Lafond D, Descarreaux M. Kinematic and electromyographic parameters of the cervical flexion-relaxation phenomenon: The effect of trunk positioning. Annals of physical and rehabilitation medicine. 2009;52(1):49-58.

16. Murphy BA, Marshall PW, Taylor $\mathrm{HH}$. The cervical flexion-relaxation ratio: reproducibility and comparison between chronic neck pain patients and controls. Spine. 2010;35(24):2103-8. 
17. Maroufi N, Ahmadi A, Khatir SRM. A comparative investigation of flexion relaxation phenomenon in healthy and chronic neck pain subjects. European Spine Journal. 2013;22(1):162-8.

18. Choi K-H, Cho M-U, Park C-W, Kim S-Y, Kim M-J, Hong B, et al. A Comparison Study of Posture and Fatigue of Neck According to Monitor Types (Moving and Fixed Monitor) by Using Flexion Relaxation Phenomenon (FRP) and Craniovertebral Angle (CVA). International Journal of Environmental Research and Public Health. 2020;17(17):6345.

19. Mousavi-Khatir R, Talebian S, Maroufi N, Olyaei GR. Effect of static neck flexion in cervical flexionrelaxation phenomenon in healthy males and females. Journal of bodywork and movement therapies. 2016;20(2):235-42.

20. Rose-Dulcina K, Genevay S, Dominguez D, Armand S, Vuillerme N. Flexion-relaxation ratio asymmetry and its relation with trunk lateral rom in individuals with and without chronic nonspecific low back pain. Spine. 2020;45(1):E1-E9.

21. Shamsi H, Khademi-Kalantari K, Akbarzade-Baghban A, Izadi N, Okhovatian F. Effect of eight weeks of cervical stability exercise on flexion relaxation phenomenon, pain, and disability in patients with non-specific chronic neck pain. 2020.

22. Yeo S-S, Kwon J-W. A Comparison study of cervical flexion-relaxation ratio in the normal and forward head postures. The Journal of Korean Physical Therapy. 2020;32(6):378-82.

23. Johnston V, Jull G, Darnell R, Jimmieson N, Souvlis T. Alterations in cervical muscle activity in functional and stressful tasks in female office workers with neck pain. European journal of applied physiology. 2008;103(3):253-64.

24. Lascurain-Aguirrebeña I, Newham DJ, Irazusta J, Seco J, Critchley DJ. Reliability of a method to measure neck surface electromyography, kinematics, and pain occurrence in participants with neck pain. Journal of manipulative and physiological therapeutics. 2018;41(5):413-24.

25. Shamsi H, Khademi-Kalantari K, Akbarzadeh-Baghban A, Izadi N, Okhovatian F. Cervical flexion relaxation phenomenon in patients with and without non-specific chronic neck pain. Journal of Back and Musculoskeletal Rehabilitation. 2021(Preprint):1-8.

26. Franke J, Hesse T, Tournier C, Schuberth W, Mawrin C, LeHuec JC, et al. Morphological changes of the multifidus muscle in patients with symptomatic lumbar disc herniation. Journal of Neurosurgery: Spine. 2009;11(6):710-4.

27. Amiri-Arimi S, Bandpei MAM, Rezasoltani A, Javanshir K, Biglarian A. Asymmetry of Cervical Multifidus and Longus Colli Muscles Size in Participants With and Without Cervical Radicular Pain. Journal of Manipulative and Physiological Therapeutics. 2020;43(3):206-11.

28. Noormohammadpour P, Dehghani-Firouzabadi A, Mansournia MA, Mohseni-Bandpei MA, Moghaddam N, Miri M, et al. Comparison of the Cross-Sectional Area of Longus Colli Muscle Between Patients With Cervical Radicular Pain and Healthy Controls. PM\&R. 2017;9(2):120-6.

29. Fortin M, Dobrescu O, Courtemanche M, Sparrey C, Santaguida C, Fehlings MG, et al. Association between paraspinal muscle morphology, clinical symptoms and functional status in patients with degenerative cervical myelopathy. The Spine Journal. 2016;16(10):S200. 
30. Park K-N, Kwon O-Y, Kim S-J, Kim S-H. Asymmetry of neck motion and activation of the cervical paraspinal muscles during prone neck extension in subjects with unilateral posterior neck pain. Journal of back and musculoskeletal rehabilitation. 2017;30(4):751-8.

31. Halvorsen M, Abbott A, Peolsson A, Dedering Å. Endurance and fatigue characteristics in the neck muscles during sub-maximal isometric test in patients with cervical radiculopathy. European spine journal. 2014;23(3):590-8.

32. Johnston V, Jull G, Souvlis T, Jimmieson NL. Neck movement and muscle activity characteristics in female office workers with neck pain. Spine. 2008;33(5):555-63.

33. Lascurain-Aguirrebeña I, Newham DJ, Galarraga-Gallastegui B, Critchley DJ. Differences in neck surface electromyography, kinematics and pain occurrence during physiological neck movements between neck pain and asymptomatic participants. A cross-sectional study. Clinical Biomechanics. 2018;57:1-9.

34. Nicholson KJ, Millhouse PW, Pflug E, Woods B, Schroeder GD, Anderson DG, et al. Cervical sagittal range of motion as a predictor of symptom severity in cervical spondylotic myelopathy. Spine. 2018;43(13):883-9.

35. Machino M, Yukawa Y, Imagama S, Ito K, Katayama Y, Matsumoto T, et al. Age-related and degenerative changes in the osseous anatomy, alignment, and range of motion of the cervical spine: a comparative study of radiographic data from 1016 patients with cervical spondylotic myelopathy and 1230 asymptomatic subjects. Spine. 2016;41(6):476-82.

\section{Tables}

Table 1: The comparison of baseline characteristics

\begin{tabular}{cccc} 
Groups & $\begin{array}{c}\text { Patients } \\
\text { Variables }\end{array}$ & $\begin{array}{c}\text { Controls } \\
(\text { mean } \pm \text { SD) }\end{array}$ & P-value \\
\hline Age (y) & $44.36 \pm 10.81$ & $44.72 \pm 10.46$ & 0.905 \\
Weight $(\mathrm{Kg})$ & $75.76 \pm 10.61$ & $72.03 \pm 7.83$ & 0.164 \\
Height (m) & $1.64 \pm 0.09$ & $1.65 \pm 0.07$ & 0.563 \\
BMI & $28.27 \pm 4.72$ & $26.37 \pm 3.28$ & 0.107
\end{tabular}

$B M I=$ body mass index

Table 2: The comparison of cervical FRR, $\triangle F R R$, and cervical ROM in sagittal plate 


\begin{tabular}{llll} 
Variables & Patients & Controls & P-value \\
\hline m.FRR & $4.30 \pm 1.4$ & $5.70 \pm 2.3$ & $.015^{\star}$ \\
\hline$\Delta$ FRR & $3.34 \pm 2.2$ & $1.74 \pm 1.1$ & $.003^{\star}$ \\
\hline CF.ROM & $40.85 \pm 6.2^{\circ}$ & $42.68 \pm 4.7^{\circ}$ & .25 \\
\hline CE.ROM & $28.08 \pm 3.9^{\circ}$ & $34.22 \pm 4.8^{\circ}$ & $.000^{\star}$
\end{tabular}

$M . F R R=$ mean flexion-relaxation ratio, $\triangle F R R=$ asymmetry of flexion-relaxation ratio between right and left sides, CF.ROM= cervical flexion range of motion, CE.ROM= cervical extension range of motion

*Significant based on the independent-samples t-test results

Table 3: The comparison of the involved and non-involved sides in the patient group

\begin{tabular}{|llll|}
\hline Patients & Involved side ES & Non-involved side ES & P-value \\
\hline FRR & $3.04 \pm 1.00$ & $4.47 \pm 1.94$ & $0.004^{*}$ \\
\hline
\end{tabular}

*Significant based on the paired-samples $t$-test results, FRR $=$ flexion relaxation ratio

\section{Figures}




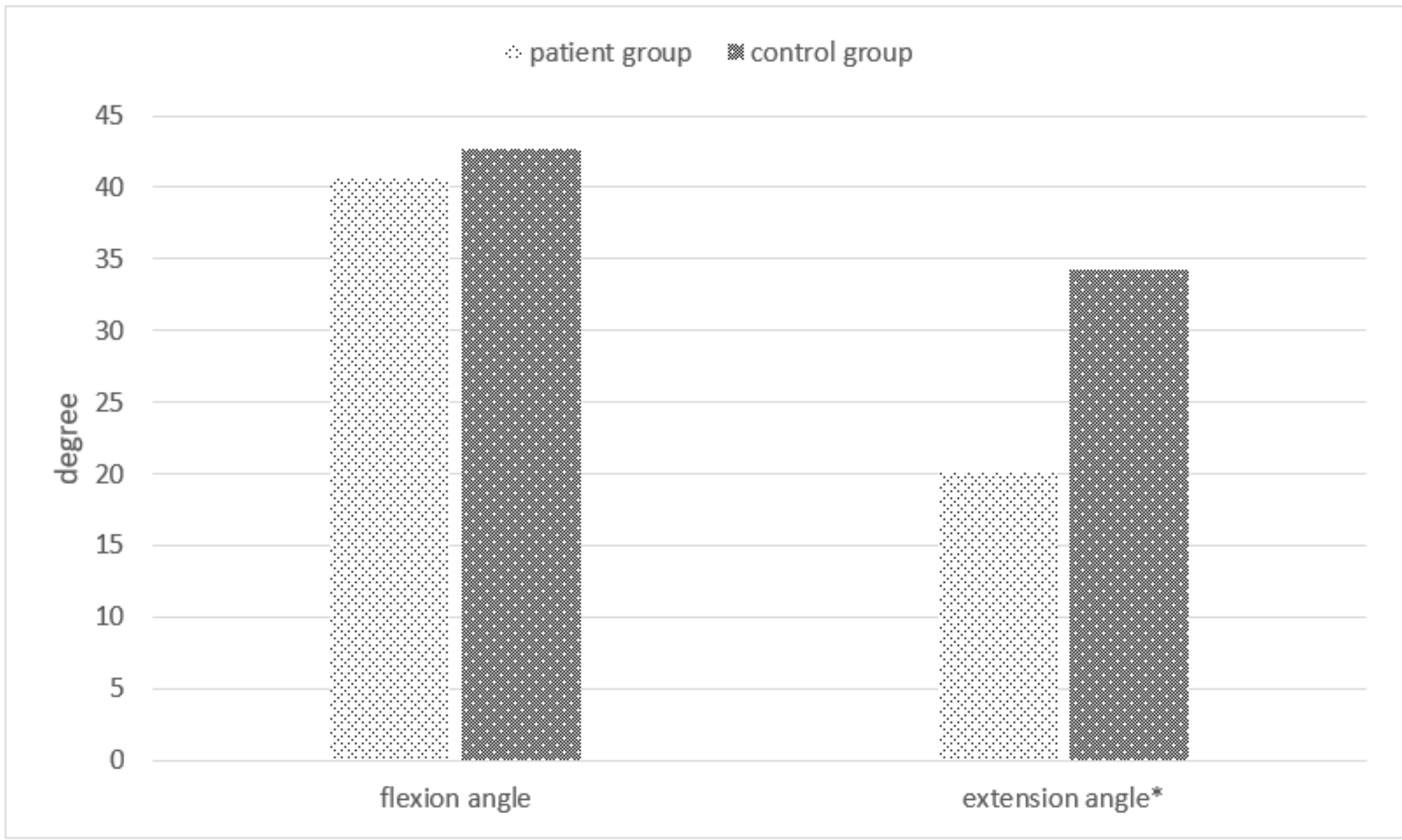

Figure 1

CF and CE angles in patients and controls

*Significant based on the independent-samples t-test results 